\title{
Efecto del procesado termomecánico sobre las propiedades mecánicas de la aleación MA956. I. Caracterización microestructural
}

\author{
J. Chao $^{(*)}$, M.C. Cristina ${ }^{(*)}$, J.L. González-Carrasco ${ }^{(*)}$ y G. González-Doncel ${ }^{(*)}$
}

\begin{abstract}
Resumen Se describen las características microestructurales y la textura de la aleación MA956 en las etapas intermedias de su procesado termomecánico para obtener el producto en forma de barras. Se partió de un material procesado en INCO Alloys International de tres formas: compactado isostáticamente, extruido en caliente $+1.330^{\circ} \mathrm{C} / 1 \mathrm{~h}$ y laminado en caliente. Los materiales procesados mediante compactación isostática y laminación en caliente, se sometieron, además, a dos tipos de recocido, uno a $1.100{ }^{\circ} \mathrm{C} / 100 \mathrm{~h}$ y otro a $1.330^{\circ} \mathrm{C} / 1 \mathrm{~h}$. En el caso del material procesado mediante extrusión en caliente, la textura inicial de fibra del tipo $<110>$ evoluciona hacia una del tipo $<111>$ cuando se la somete al tratamiento de $1.330^{\circ} \mathrm{C} / 1 \mathrm{~h}$, mientras que en el caso del material procesado mediante laminación en caliente, la textura inicial, con componentes $\langle 110\rangle$ y $\langle 100\rangle$, cambia hacia una del tipo $<100>$ después del mismo tratamiento. La razón de la diferencia en la textura final del material procesado por ambos métodos se atribuye a las diferencias en la fracción en volumen de granos primarios con orientaciones $<110>$ y $<100>$.
\end{abstract}

Palabras clave: Procesado termomecánico. Tratamiento térmico. Microestructura. Aleación MA956

\section{Effect of the thermomechanical processing on the mechanical properties of MA956 alloy. I. Microstructural characterization}

\begin{abstract}
Both the microstructural characteristics and the texture of the ferritic oxide dispersion strengthened MA956 alloy have been studied after different stages of processing. The material was supplied by INCO Alloys International in three conditions: hot isostatically pressed (HIP), hot extruded $+1,330$ ${ }^{\circ} \mathrm{C} / 1 \mathrm{~h}$ and hot rolled. Two annealing treatments: $1,100{ }^{\circ} \mathrm{C} / 100 \mathrm{~h}$ and $1,330{ }^{\circ} \mathrm{C} / 1 \mathrm{~h}$ were performed on the HIP and hot rolled materials to observe the effect of previous microstructure (or the thermomechanical history) on the final microstructure. In the hot-extruded condition, the initial strong $\langle 110\rangle$ fibre texture evolves towards a very strong $<111\rangle$ texture after $1,330^{\circ} \mathrm{C} / 1 \mathrm{~h}$ treatment, whereas in the hot rolled condition the initial fibre texture with components $\langle 110\rangle$ and $<100\rangle$ results in $a<100>$ after the same thermal treatment. It is pointed out that this difference in the final texture could be due to the differences in the volume fraction of primary grains with $<100>$ and $<111>$ orientations.
\end{abstract}

Keywords: Thermomechanical processing. Heat treatment. Microstructure. MA956 alloy.

\section{INTRODUCCIÓN}

La aleación MA956 es un material diseñado para trabajar a temperaturas de servicio elevadas. Por esta razón, tanto el método de fabricación (aleado mecánico) como su posterior tratamiento termo-

(*) Centro Nacional de Investigaciones Metalúrgicas, CENIM (CSIC), Avda. de Gregorio del Amo, 8. 28040-Madrid (España). mecánico, están ideados para conferir al material características microestructurales óptimas para trabajar a altas temperaturas. Una de estas características es una microestructura con una baja densidad de fronteras de grano. De esta forma, se consigue incrementar la resistencia a la deformación por deslizamiento de fronteras de grano. La resistencia al movimiento de dislocaciones se eleva en virtud de una distribución de dispersoides muy finos de óxidos de itrio (1), los cuales, por otra parte, son estables hasta temperaturas próximas a $\operatorname{los} 1.300{ }^{\circ} \mathrm{C}$ 
(2). La textura desempeña un papel importante, ya que debe ser tal que las propiedades mecánicas de la aleación en la dirección en la que van a ser utilizadas en servicio sean las óptimas. Esta aleación tiene, por último, la capacidad de generar, mediante un simple proceso de oxidación térmica, una capa de alúmina densa y muy bien adherida al substrato que la protege de ulterior oxidación cuando se somete a altas temperaturas de servicio. Se ha comprobado, además, que a temperatura ambiente, dicha capa de alúmina, mejora considerablemente la resistencia a la corrosión de la aleación frente a fluidos orgánicos (3), siendo ésta incluso superior a la de los biomateriales en uso (4).

En esta primera parte, se trata de analizar cómo las sucesivas etapas del procesado termomecánico de la aleación MA956 en forma de barras, influyen en sus características microestructurales.

\section{ANTECEDENTES DE LA ALEACIÓN Y DETALLES EXPERIMENTALES}

La composición química nominal de la aleación es $20 \mathrm{Cr}-4,5 \mathrm{Al}-0,5 \mathrm{Ti}-0,5 \mathrm{Y}_{2} \mathrm{O}_{3}$ (\% en masa), resto hierro. El proceso de fabricación (esquematizado en la figura 1) del producto en forma de barras es el siguiente (5): Las partículas de hierro, de aleación madre y de itria, se someten a un proceso de aleado mecánico en un molino de bolas, dando lugar, después de unas $10 \mathrm{~h}$, a unas partículas de tamaño comprendido entre 50 y $150 \mu \mathrm{m}$ (6). La microestructura de estas partículas tiene un tamaño de grano inferior a la micra. Estas partículas se encap-

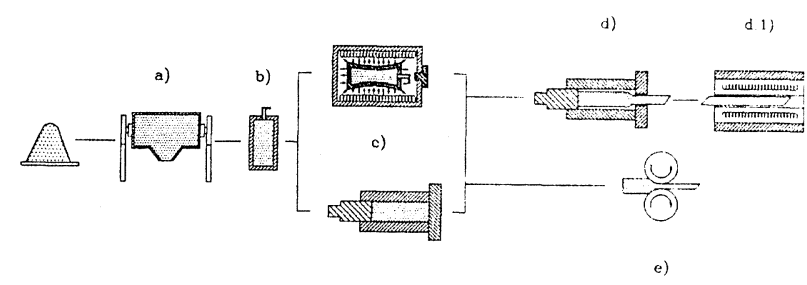

FIG. 1.- Esquema del proceso de fabricación de barras de la aleación MA956. a) Aleado mecánico. b) Encapsulado y desgasificación. c) Consolidación isostática o uniaxial a $1.000{ }^{\circ} \mathrm{C} . d$ ) Extrusión con una relación de $20: 1$ a $1.000-1.050{ }^{\circ} \mathrm{C}$. $d I$ ) Tratamiento de recristalización a $1.330{ }^{\circ} \mathrm{C} / 1 \mathrm{~h}$. e) Laminación a $1.100{ }^{\circ} \mathrm{C}$ para barras de diámetro $<16 \mathrm{~mm}$.

FIG. 1.- Scheme of the fabrication route for MA 956 bars: a) Mechanical Alloying. b) Canning and degassing. c) Isostatic or uniaxial consolidation at about 1,000 ${ }^{\circ} \mathrm{C}$. d) Extrusion at 20:1 and 1,000$1,050^{\circ} \mathrm{C}$. dl) Recrystallization treatment at 1,330 ${ }^{\circ} \mathrm{C} / 1$ h. e) Rolling at $1,100{ }^{\circ} \mathrm{C}$ for bars $<16 \mathrm{~mm}$ in diameter. sulan en un recipiente de acero suave y se compactan, uniaxial o isostáticamente a $1.000{ }^{\circ} \mathrm{C}(6)$. A continuación, se procede a su extrusión con una relación de $20: 1$, a $1.000-1.050{ }^{\circ} \mathrm{C}$, y posteriormente se recristaliza a $1.330^{\circ} \mathrm{C}(6$ y 7$)$. Según el fabricante (INCO Corp.), el conformado de las barras de un diámetro menor de $16 \mathrm{~mm}$ no se realiza mediante extrusión, sino por laminación a $1.100{ }^{\circ} \mathrm{C}$, aplicando un grado de reducción del $95 \%$ (8).

La aleación la suministró el fabricante en tres estados, correspondientes a las diferentes etapas de procesado del material:

- Compactada isostáticamente en forma de cilindros de $80 \mathrm{~mm}$ de diámetro.

- Extruida en caliente y recristalizada a $1.330{ }^{\circ} \mathrm{C}$ durante $1 \mathrm{~h}$ en forma de barras de $60 \mathrm{~mm}$ de diámetro.

- Laminada en caliente, en forma de barras de 9,5 mm de diámetro.

En los casos de los materiales extruido y laminado, la consolidación previa de los polvos se llevó a cabo mediante compactación uniaxial en la prensa de extrusión.

En los materiales laminado y compactado isostáticamente, se realizaron dos tipos de tratamiento: $a$ ) Recocido a $1.330{ }^{\circ} \mathrm{C}$ durante $1 \mathrm{~h}$. Este tratamiento se realizó con objeto de analizar el efecto del conformado del material sobre sus propiedades. $b$ ) Recocido a $1.100{ }^{\circ} \mathrm{C}$ durante $100 \mathrm{~h}$, que es el tratamiento para generar la capa de alúmina.

La caracterización microestructural se llevó a cabo mediante microscopía óptica y electrónica de barrido. La textura de los materiales se obtuvo mediante difracción de rayos $\mathrm{X}$ en un anillo de Euler cerrado y se analizó a partir de la figura de polos y la función de distribución de orientaciones (FDO).

\section{RESULTADOS Y DISCUSIÓN}

\subsection{Material compactado isostáticamente}

La microestructura del material antes de ser sometido a algún tratamiento térmico, consiste en granos equiaxiales con un diámetro medio próximo a $1 \mu \mathrm{m}$. Dentro de estos granos, se encuentran distribuidos uniformemente dispersoides de ytria de unos $10 \mathrm{~nm}$ de diámetro y un interespaciado de aproximadamente $100 \mathrm{~nm}$ (2). Por la naturaleza del conformado, los granos no poseen una orientación cristalina preferente, es decir, no tiene textura. El tratamiento térmico a $1.100{ }^{\circ} \mathrm{C} / 100 \mathrm{~h}$ da lugar a una microestructura con un tamaño de grano de $10 \mu \mathrm{m}$ (Fig. 2a). El tratamiento a $1.330{ }^{\circ} \mathrm{C} / 1 \mathrm{~h}$ produce un crecimiento considerable en el diámetro medio de grano (Fig. 2b), llegando ocasionalmente 

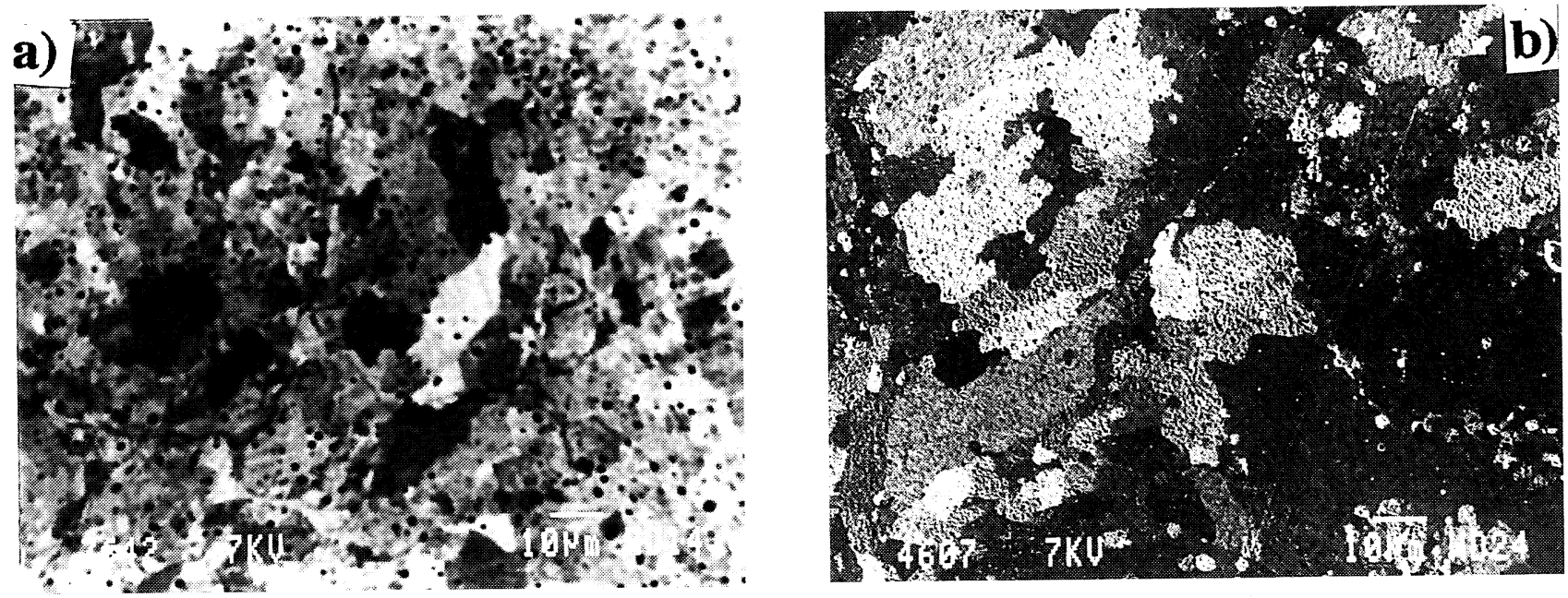

FIG. 2.- Microestructura del material compactado isostáticamente después del tratamiento térmico. a) $1.100{ }^{\circ} \mathrm{C} / 100$ h. b) $1.330{ }^{\circ} \mathrm{C} / 1 \mathrm{~h}$.

FIG. 2.- Microstructure of the hot isostatic pressed material after heat treatments at: a) $1,100{ }^{\circ} \mathrm{C} / 100 \mathrm{~h}$, and b) $1,330{ }^{\circ} \mathrm{C} / \mathrm{lh}$.

a igualarse al de la partícula (hasta $150 \mu \mathrm{m})$ procedente del aleado mecánico.

\subsection{Material extruido}

Los granos son muy alargados y entrelazados en la dirección de extrusión. Sus dimensiones medias son: $0,4 \mu \mathrm{m}$ de diámetro y $5 \mu \mathrm{m}$ de longitud (7). La textura, medida en diferentes posiciones sobre un plano diametral de la barra es muy nítida, del tipo fibra $<110\rangle$ en la dirección longitudinal de la barra, lo cual está en consonancia con otras investigaciones (7 y 9).

Cuando se le somete al tratamiento a $1.330{ }^{\circ} \mathrm{C}$ durante $1 \mathrm{~h}$ se produce un crecimiento espectacular del grano, como se observa en la fotomacrografia de la sección transversal en la figura 3. El material extruido y recristalizado también presentaba una marcada textura de fibra, esta vez con el eje de la barra en la dirección $\langle 111\rangle$, lo cual también coincide con los resultados de trabajos anteriores (7).

\subsection{Material laminado}

La forma de los granos antes de cualquier tratamiento térmico es muy similar a la del material extruido antes de recristalizar. Es de esperar, sin embargo, que el tamaño de grano sea algo mayor que en el material extruido, dado que la temperatura de conformación es ligeramente superior. A diferencia del material extruido (textura $<110>$ ), el material laminado presenta una textura de fibra con dos componentes: $<110\rangle$ y $<100\rangle$. En la figura 4 se representa la intensidad máxima de dichas compo- nentes en función de la distancia al centro de la barra. Como se observa, la componente $<100>$ aumenta conforme se aproxima a la superficie de la barra, mientras que la $\langle 110\rangle$ disminuye. En el centro de la barra, la intensidad máxima de la componente $\langle 100\rangle$ es aproximadamente el doble de la $<110\rangle$, mientras que en la superficie son igualmente intensas. El tratamiento térmico a $1.100^{\circ} \mathrm{C} / 100 \mathrm{~h}$

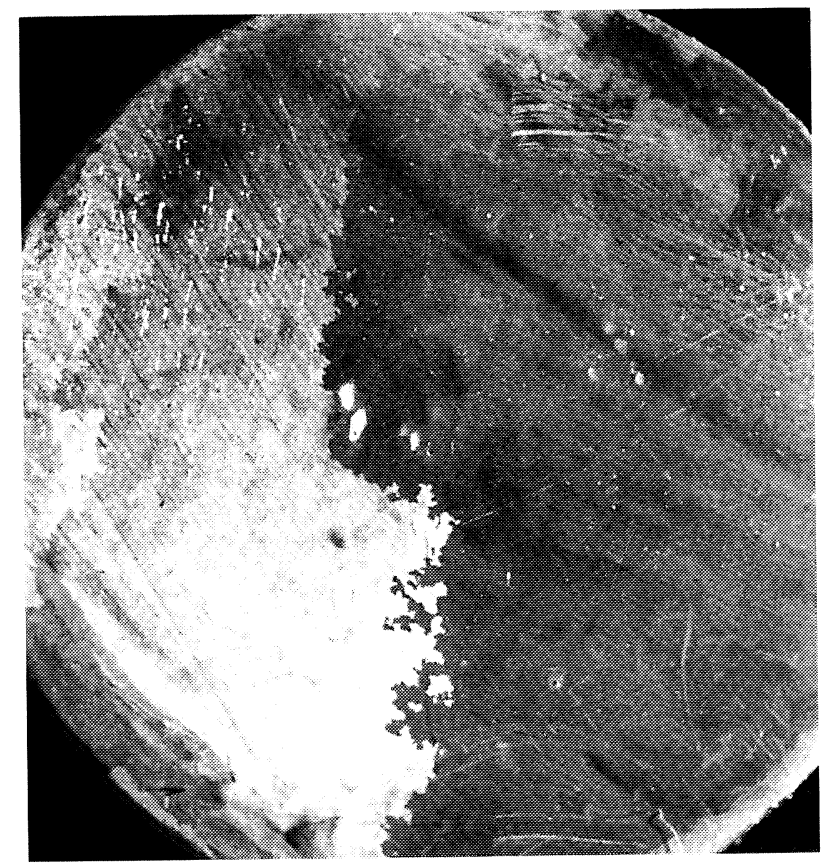

FIG. 3.- Fotomacrografia de la sección transversal de la barra del material extruido después del tratamiento térmico a $1.330{ }^{\circ} \mathrm{C} / 1 \mathrm{~h} . \times 7$.

FIG. 3.- Macrograph of a cross section of the extruded bar after heat treatment at $1,330^{\circ} \mathrm{C} / 1 \mathrm{~h} . \times 7$. 


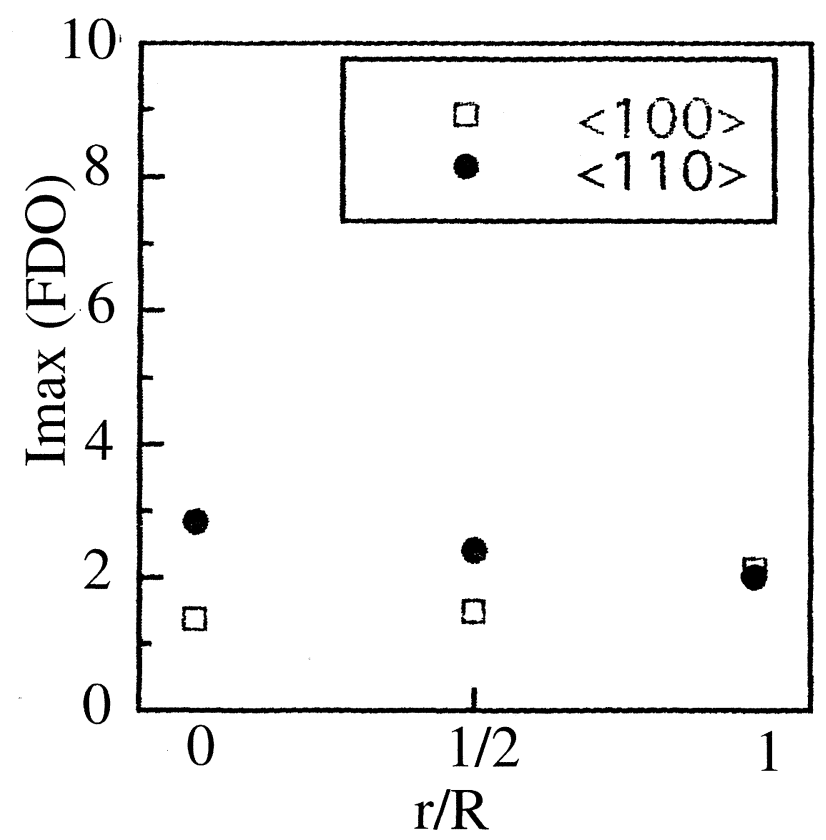

FIG. 4.- Variación con la distancia al centro de la barra de la intensidad máxima de las componentes principales de la textura del material laminado.

FIG. 4.- Variation of the maximum intensity of the main texture components of the rolled material as a function of the distance to the centre of the bar.

modifica la microestructura en dos aspectos. En primer lugar, el tamaño de grano crece en el sentido longitudinal de la barra (Fig. 5a), llegando a alcanzar hasta unos $20 \mathrm{~mm}$ de longitud y un diámetro medio de unas $150 \mu \mathrm{m}$. En segundo lugar, la textura del tipo $<100>$ se hace mucho más acusada que la $<110>$, particularmente en la superficie de la barra (Figura 5b). Para estudiar con más detalle la evolución de la textura durante el tratamiento, se realizaron tratamientos con tiempos de exposición de 3 y $6 \mathrm{~min}$, empleando una velocidad tanto de calentamiento como de enfriamiento de $50{ }^{\circ} \mathrm{C} / \mathrm{s}$ sobre muestras extraídas de la barra de $2 \mathrm{~mm}$ de espesor, $9 \mathrm{~mm}$ de ancho y $12 \mathrm{~mm}$ de longitud. En la figura $5 \mathrm{~b}$ se encuentra representada la variación con la distancia al centro de la barra de la intensidad máxima de las componentes $<110>$ y $<100>$ para dichos tiempos de tratamiento. Dicha figura pone de manifiesto que el proceso de recristalización se inicia a partir de la zona central y hacia la superficie entre los 3 y 6 min de tratamiento térmico.

El tratamiento a $1.330{ }^{\circ} \mathrm{C} / 1 \mathrm{~h}$ da lugar a una microestructura con una textura muy similar a la del material laminado y tratado a $1.100^{\circ} \mathrm{C} / 100 \mathrm{~h}$, es decir, una textura de fibra $\langle 100\rangle$. Los granos presentan un aspecto muy alargado y un diámetro medio de $300 \mu \mathrm{m}$ (Fig. 6).

El desarrollo de la microestructura en forma de granos de gran diámetro y extraordinariamente alar-
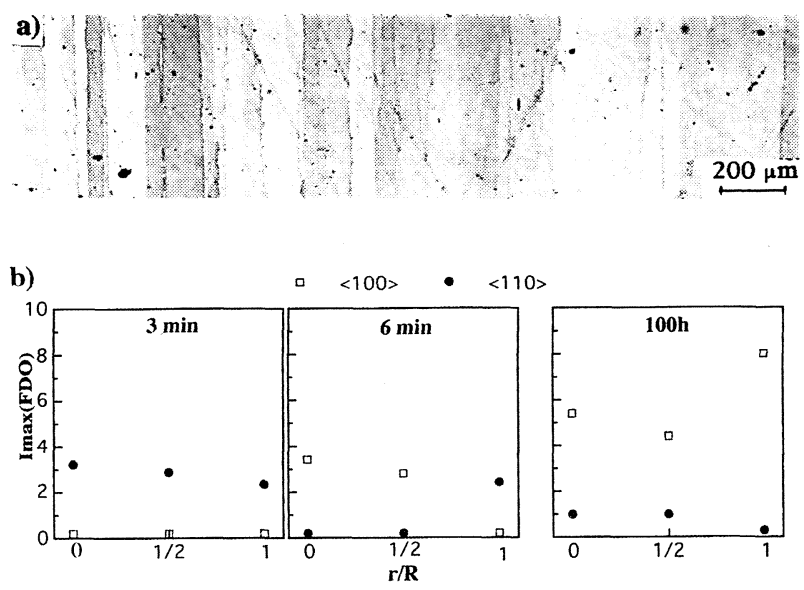

FIG. 5.- Microestructura de la barra laminada tratada a $1.100{ }^{\circ} \mathrm{C}$. a) Aspecto de los granos, sobre un plano longitudinal a la barra, al cabo de $100 \mathrm{~h}$ de tratamiento. b) Intensidad máxima de las componentes de la textura $<110>$ y $<100>$ en función de la distancia al centro de la barra después de $3 \mathrm{~min}, 6 \mathrm{~min}$ y $100 \mathrm{~h}$ de tratamiento térmico.

FIG. 5.-Microstructure of the rolled bar after heat treatment at $1,100^{\circ} \mathrm{C}$. a) Aspect of the grains on a plane parallel to the longitudinal direction of the bar after $100 \mathrm{~h}$ of heat treatment. b) Maximum intensity of the $\langle 110\rangle$ and $\langle 100\rangle$ texture components as a function of the distance to the centre of the bar after $3 \mathrm{~min}, 6 \mathrm{~min}$ and $100 \mathrm{~h}$ of heat treatment.

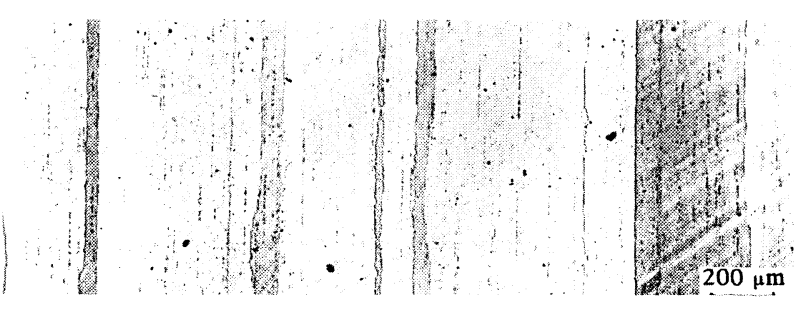

Fig. 6.- Aspecto de los granos de la barra laminada después del tratamiento a $1.330^{\circ} \mathrm{C} / 1 \mathrm{~h}$.

FIG. 6.- Grains morphology of the rolled bar after heat treatment at $1,330{ }^{\circ} \mathrm{C} / 1 \mathrm{~h}$.

gados en la dirección longitudinal de la barra es una cuestión aún controvertida, que ha sido objeto de estudios detallados de microscopía electrónica de transmisión. No obstante, es posible atribuir este fenómeno a dos aspectos fundamentales de la microestructura. El primero, que controlaría el tamaño de grano, estaría relacionado con el hecho de que el proceso de conformado produce una microestructura con una densidad uniforme de dislocaciones (6) y una textura muy acusada o, equivalentemente, una microestructura con unas fronteras de grano con un ángulo de desorientación pequeño. Una microestructura con estas características haría que el número de núcleos de recristalización fuera 
pequeño y que se produjera una microestructura con un gran tamaño de grano durante la recristalización. El segundo, que influiría en la forma alargada del grano, estaría relacionado con las cadenas de dispersoides de itria y de partículas de alúmina, que se forman debido a su segregación durante la etapa del aleado mecánico y a su posterior alineación durante las etapas de laminación ó extrusión (7). Dichas cadenas de partículas ejercerían, durante la recristalización secundaria del material, una mayor resistencia al movimiento de las fronteras de grano en la dirección transversal que en la dirección longitudinal, dando lugar, así, a la forma alargada de los granos.

La evolución de la textura desde la $<110>$ del material extruido hacia la textura $<111>$ y de las orientaciones $<110>$ y $<100>$ del material laminado hacia la textura $\langle 100\rangle$ durante el tratamiento de $1.330^{\circ} \mathrm{C} / 1 \mathrm{~h}$, es una consecuencia de un proceso de recristalización secundaria. Dicho proceso, en el caso de aleaciones ODS ferriticas, está controlado por la textura (7) y por el tamaño de grano iniciales (10). Además, ambos factores son muy sensibles a ligeras variaciones en el tratamiento termomecánico previo. El hecho de que el material posea una textura del tipo $<110>$ muy marcada hace que las fronteras de granos aislados con orientaciones cristalinas $<111>$ ó $<100>$ tengan una gran movilidad con relación a la mayoría de los granos con orientación cristalina $<110\rangle$. Esto favorece que los primeros crezcan a expensas de estos últimos. Este mecanismo se potenciaría aún más con un tamaño de grano inicial muy fino, ya que, entonces, los dispersoides inhibirían el crecimiento de los granos primarios. Recientemente (11) se ha propuesto que esta acción inhibidora la llevan a cabo los propios vértices del grano primario. La diferencia observada en la textura de los materiales laminado y extruido después del tratamiento térmico a $1.330{ }^{\circ} \mathrm{C} / 1 \mathrm{~h}$ se podría atribuir a la mayor o menor densidad de núcleos de recristalización secundaria con orientaciones cristalinas $<111>$ ó $<100>$.

\section{Agradecimiento}

Este trabajo ha sido financiado por la CICYT. Proyecto MAT95-0796.

\section{REFERENCIAS}

(1) Dubiel, B., Osuch, W., Wrobel, M., CzyrskaFilemonowicz, A. y ENNIS, P.J. Proc. Conf. Materials for Advanced Powder Engineering. Part II. Ed. por D. Coutsouradis, J.H. Davidson, J. Ewald, P. Greenfield, T. Khan, M. Malik, D.B. Meadowcroft, V. Regis, R.B. Scarlin, F. Schubert y D.V. Thornton, 1994. Kluwer Academic Press. 1.523.

(2) Cama, H. y Hughes, T.A. idem, 1.497.

(3) Escudero, M.L. y Gonzalez-CARrasco, J.L. Biomaterials, 15, 1994: 1.175.

(4) Escudero, M.L., Gonzalez-Carrasco, J.L., GarciaAlonso, M.C. y Ramirez, E. J. Biom. Mat. Res., 31, 1996: 313.

(5) Publ., IAI-9-2, de INCO. 1993.

(6) Klug, R.C., Krauss, G. y Matlock, D.K. Metall. Mater. Trans., 27A, 1996: 1945.

(7) Evens, P.J., Martin, J.W. y Little, E.A. Mater. Sci. Technol., (8), 1992: 531.

(8) Gessinger, G.H. y Mercier, O. Powder Metall. Int., 10, 1978: 202.

(9) Alamo, A., Regle, H., Pons, G. y Bechade, J.L. Mater. Sci. Forum, 88-90, 1992: 183.

(10) Sporer, D. y Korb, G. Mechanical Alloying for Structural Applications. ASM International. Materials Park, (OH, EE.UU.), 1993: 109

(11) BhadeShiA, H.K.D.H. Mater. Sci. Eng., A223, 1997: 64. 